\title{
Coordinated Analyses of a Supernova Silicate Grain in the CO3.0 Chondrite Miller Range 07687
}

Laura Seifert ${ }^{1}$, Pierre Haenecour ${ }^{2}$, Tarunika Ramprasad ${ }^{3}$ and Thomas Zega ${ }^{2}$

${ }^{1}$ Lunar and Planetary Laboratory, University of Arizona, Tucson, Arizona, United States, ${ }^{2}$ Lunar and Planetary Laboratory, University of Arizona, United States, ${ }^{3}$ Materials Science and Engineering, University of Arizona, Tucson, Arizona, United States

Supernovae are extreme explosions that occur near the end of a massive star's lifetime when it runs out of nuclear fuel, undergoes a core-collapse, and expels material through a radial explosion. Solid dust grains can condense in this ejected material. A fraction of such grains survived transport through the interstellar medium and were incorporated into primitive solar system materials including meteorites, micrometeorites, interplanetary dust particles, and comet samples returned by the NASA Stardust mission [e.g. 1]. Analysis of supernova (SN) grains can provide ground-truth information on the chemical and thermodynamic conditions in the ejecta of stellar explosions, information that is otherwise inaccessible through remote observation. Here we discuss the detailed analysis of a SN silicate grain using a coordinated analytical approach that includes nanoscale secondary ion mass spectrometry (NanoSIMS), focused-ion beam scanning-electron microscopy (FIB-SEM), and transmission electron microscopy (TEM).

Isotopically anomalous SN grains were identified via raster-ion-imaging in a petrographic thin section of the CO 3.0 chondrite Miller Range (MIL) 07687 at Washington University in St. Louis [2]. We selected one grain, 'MIL 2a7-25 o1' from an unaltered region of MIL 07687 [2] for detailed analysis using TEM. An electron transparent cross-section of MIL 2a7-25 o1 was prepared using the FEI Helios G3 FIB-SEM located at the Lunar and Planetary Laboratory (LPL) using well-established techniques [3]. Prior to FIB extraction, isotopic data was correlated with secondary electron images to confirm the location of the isotopic anomaly ('hotspot'). We placed Pt fiducial markers over the hotspot to protect the area from the ion beam and mark the location of the anomalous grain. A C strap was deposited on top of the Pt fiducial markers and extending the full length of the section $(\sim 6 \mu \mathrm{m})$ to protect the region of interest from $\mathrm{Ga}^{+}$implantation and amorphization. The FIB section was then thinned to electron transparency $(<100 \mathrm{~nm})$ and analyzed using the Hitachi HF5000 scanning TEM (S/TEM) at $200 \mathrm{keV}$ located at LPL. The HF5000 is equipped with a cold FEG, $3{ }^{\text {rd }}$-order spherical aberration corrector for STEM mode, STEM-based secondary electron (SE), bright-field (BF), and dark-field (DF) imaging detectors, as well as an Oxford Instruments X-Max ${ }^{\mathrm{N}} 100$ TLE energy dispersive X-ray spectroscopy (EDS) system with dual $100 \mathrm{~mm}^{2}$ windowless silicon-drift detectors (solid angle of $2 \mathrm{sr}$ ).

NanoSIMS analysis reveals that MIL 2a7-25 o1 contains enrichments in ${ }^{18} \mathrm{O}$ relative to solar system values with ${ }^{18} \mathrm{O} /{ }^{16} \mathrm{O}\left(\mathrm{x} 10^{-3}\right)=3.34 \pm 0.07$ and ${ }^{17} \mathrm{O} /{ }^{16} \mathrm{O}\left(\mathrm{x} 10^{-4}\right)=3.92 \pm 0.23$, placing it in the Group-4 field of presolar grains as defined by [4]. STEM imaging reveals that the domain beneath the Pt fiducial marker consists of fine-grained ( 10 to $60 \mathrm{~nm})$ material (Fig. 1a-b). STEM-EDS maps $(512 \times 512 \mathrm{px})$ show that the hotspot located below the Pt fiducial marker contains $\mathrm{O}, \mathrm{Si}, \mathrm{Mg}$ and $\mathrm{Fe}$, with local Fe enrichment near the center of MIL 2a7-25 o1 (arrow, Fig. 1e). Selected-area electron-diffraction (SAED) patterns acquired across the hotspot reveal that MIL 2a7-25 o1 is polycrystalline. Measurements of SAED patterns reveal d-spacings that overlap with both olivine and pyroxene. It is therefore difficult to pinpoint a single-phase carrier of the isotopic anomaly corresponding to MIL 2a7-25 o1. We consider the entire polycrystalline assemblage below the Pt marker as carrying the isotopic anomaly and hence as the circumstellar 'grain'.

MIL 07687 contains mixed domains of altered and unaltered material [2]. This FIB section was extracted from an unaltered region, and we do not observe evidence that might otherwise suggest that secondary processing altered MIL 2a7-25 ol. Thus, as a working hypothesis, we rule out secondary processing but plan to test it 
further via comparison with FIB sections from altered regions. Given that MIL 2a7-25 o1 is polycrystalline, precisely constraining the exact conditions under which it formed is difficult. Polycrystalline and amorphous aggregates of circumstellar grains were previously reported [5-8] and are thought to suggest nonequilibrium or multistep condensation processes. We do not observe amorphous grains in or around the MIL 2a7-25 o1 assemblage that could suggest nonequilibrium condensation, therefore a multistep formation process is consistent with the data. Equilibrium models of dust condensation in SNe predict orthopyroxene to condense between 1090 and $1548 \mathrm{~K}$ and olivine to condense between 1063 and $1575 \mathrm{~K}$ [9]. As temperatures cooled in the SN ejecta, it is possible that nanocrystalline olivine and pyroxene condensed in a stepwise manner and mechanically accreted to form the assemblage we observe.
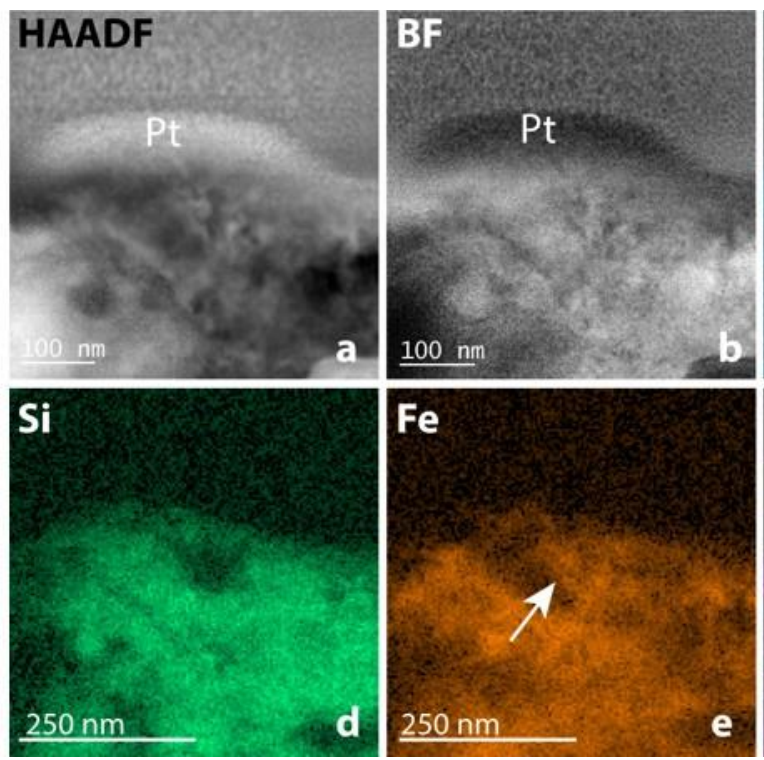
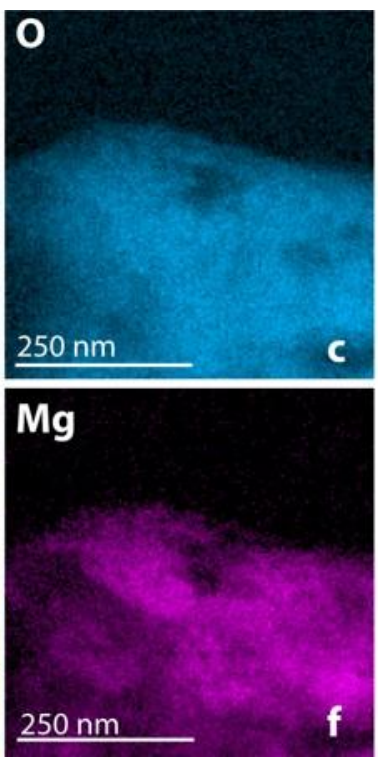

Figure 1. a-b) HAADF and BF images of MIL 2a7-25 o1 below Pt fiducial marker. c-f) False color EDS maps of MIL 2a7-25 o1 for $\mathrm{O}, \mathrm{Si}, \mathrm{Fe}$, and $\mathrm{Mg}$, respectively.

\section{References}

[1] Zinner, E. in “Treatise on Geochemistry”, Elsevier Ltd (2014), p. 181.

[2] Haenecour, P. et al., M\&PS, 55 (2020), p. 1228.

[3] Zega, T. J. et al., M\&PS, 42 (2007), p. 1373.

[4] Nittler, L. et al., ApJ, 483 (1997), p. 475.

[5] Stroud, R. M. et al., LPSC, 40 (2009), p. 1063.

[6] Vollmer, C. et al., ApJ, 700 (2009), p. 774.

[7] Nguyen, A. N. et al., ApJ, 719 (2010), p. 166.

[8] Zega, T. J. et al., M\&PS, 55 (2020), p. 1207.

[9] Fedkin, A. V. et al., GeCoA, 74 (2010), p. 3642.

[10] The research completed here was supported by NASA grant 80NSSC19K0509. 